\title{
A Refection on Digitalized Creation of Chinese Internet Big Movies in the Context of Internet
}

\author{
Liang Lei, Kunhao Ni, Ying Cui \\ Faculty of Arts and Communication, Kunming University of Science and Technology, Kunming 650500, Yunnan, China \\ DOI: $10.32629 /$ asc.v2i4.530
}

\begin{abstract}
The 21st century is the era of the Internet, where movies see new vitality in artistic creation under the concept of the Internet+. Internet Big movies, a new type of films born with the advancement of Internet technology, have seen rapid development in less than a decade, having gradually established a mature production model. This paper provides reflection and analysis on the new phenomena in Chinese film production that involve numerous applications of big data technologies. Keywords: Internet, Internet big movies, big data, creation, production
\end{abstract}

2014 is the year marking the first all-out push of the Internet giants into the film and television markets. A number of Chinese Internet companies, representatively including Baidu, Alibaba and Tencent, have greatly accelerated their pace in making their deployments in the film industry through financing, mergers and acquisitions, as well as strategic cooperation, directly impacting and changing the pattern of the traditional film industry ${ }^{[1]}$. In the same year, iQIYI first put forward the concept of "Internet big movie", which differs from theater-chain-based movie by featuring the Internet and from micro movie by the word "big." Internet big movies are more like a film production reform triggered by the media, just like the transition from silent films to sound films, from black-and-white films to color films, and from 2D films to today's multidimensional films. The introduction of the "Internet +" concept into the film industry, which fundamentally subverts the rules of the traditional film market in China, has provided new possibilities for the development of the film industry.

Marshall McLuhan argued that "the content of any medium is another medium, and the content of writing is language, just as the content of printing is writing, and the content of telegraph is printing." We are living in an era of multi-media integration, and Internet big movies are a mix of "Internet +" and video art. The content of an Internet big movie is the film. It is the exploration and evolution of films through modern cultures and media.

The number of Internet users in China reached 989 million as of December 2020, an increase of 85.4 million over March 2020, with the Internet penetration rate reaching $70.4 \%{ }^{[2]}$. Meanwhile, the number of subscribers of iQIYI, one of the three major synchronous platforms, reached 107 million in 2019, with the revenue of iQIYI's subscriber sector increased by $36 \%{ }^{[3]}$. Internet big movies have become a new force in the spotlight, and have gradually established a mature production model, thanks to the expansion of the market, a massive input of funds, the development of the Internet, as well as the increasing maturity of the media environment. With numerous applications of big data in the creation of Chinese Internet big movies, the following new phenomena related to movie have emerged:

\section{The creation of intellectual property (IP) under the investment model featuring a small financial input, quick revenue generation and a short investment cycle}

Internet big movies feature a small financial input, quick revenue generation and a short investment cycle, which naturally make them attractive to investors. Thus a large amount of capital has flown in and promoted the development of the Internet big movie industry. In most cases, an Internet big movie requires an investment of only millions, far less than that for a traditional theater-chain-based movie, which requires at least dozens of millions. In addition, for a theater-chain-based movie, it takes about six to twelve months to get the revenue of the investment, but for an Internet big movie, the time can be shorten to 3 months in the best case. Internet big movies are also more advantageous than theater-chain-based movies in terms of the time required for production, as the former type takes only a few months while the latter usually needs more than one year. Due to a small investment and a shorter time frame for polishing the script and producing the film, Internet big movies are prone to be short of creativity and novelty. Thus the investors have no choice but to choose intellectual properties (IPs) that can be quantified through big data screening in order to minimize the investment risk.

Many Internet big movies have been produced under the head IP model: they were intended to cause an effect of topic, or did imitations of mainstream value, so as to stand out among tons of content on Internet platforms. On the one hand, 
the nostalgia effect of a big IP can secure a certain number of fans; on the other hand, linking the film industry chain to IPs can promote the development of other relevant contents and derivative products, which can bring in more revenue. For instance, Mojin: Raiders of the Wu Gorge (2019), a classic IP-based film that ranked No. 1 at the box office in 2019, won more income from ticket sales with a lower advertising cost, thanks to the fans and popularity of the IP. Meanwhile, the "IP + star" model, where stars are used as a selling point to increase income with the help of the fans, have become necessary for some Internet big movies. These IP-based adaptations have been successful in terms of both ticket sales and popularity. Some even have been made into a film series with more related contents developed, "becoming a brand of Internet movie" [4]. Nearly 20 episodes of the Mojin series have been made into film and television works since 2014, with nearly 10 still in production ${ }^{[5]}$. "Brand is an element that may help the potential audiences to avoid making bad choices, as the audiences can identify their preferred content through brand elements such as IP. Especially, when an IP-based film has been developed into a film series, its branding and the leading role as a brand will spread its influence to the broader cultural industry." [6]. However, the explosive growth of IP-based films also has exposed a series of issues. The bubblization of IPs has reduced the confidence of many users in IP-based films. For instance, iPartment (2018), a head-IP-based big movie (not an Internet big movie) released in 2018, though with tons of fans, ended up with both poor audience feedback and ticket sales. If an Internet big movie, though with the purpose of avoiding risks and precisely securing target audiences, relies too much on IP and the popularity of stars that are suggested by big data analysis, opposite results would be seen in most case. Excessive dependence on big data would reduce the weight of the voice from creators, and only leads to a bewildering situation in the flood of data.

\section{The work system centering on Internet-based investor}

"For Internet big movies, the industrial chain mainly includes three subjects: the content producer, content operator and end user." ${ }^{[7]}$ As the hub connecting the three subjects, the Internet platform possesses massive user resources and user data, and has become the key to realizing the commercial value of Internet big movies. The Internet platform is the main funder of Internet big movies, and "Internet plus" is a common way of thinking for it, while big data analysis serves as an objective tool for prediction. At present, there are mainly two ways for big data to intervene in film creation. In the first way, a purely commercial movie is produced solely according to the principles suggested by big data analysis, which identifies the demands of target users from massive data, and then affect and guide the production of the film according to these demands. What is more, in the first way, the creation of the script, the selection of actors, and art style of the film largely depend on the results of network big data analysis. In the second way, the film production company, based on the tradition of process film production, analyzes and evaluates many elements and various links in film production, conducts research and data analysis with the help of Internet technology, and finally adjusts and determines the market positioning of the film projects and the focus of film creation." ${ }^{,[8]}$ With the intervention of big data, the Internet-based investor is inherently dominant, thus reducing the film director's power to control the film project. As the decision-maker and manager of the project, the Internetbased investor has the highest power in creation, pushing related film creators to a weak position. This in fact is the vertical monopoly in the "production, distribution and screening" of Internet big movies. In such a context, Internet big movies are characterized by taking the Internet-based investor as the core.

By analyzing the audience's preferences and viewing habits, the Internet platform screens out the contents and theme in line with the audience's needs, and construct stories based on the broadcast data. According to the market demands suggested based on the data of the platform, the Internet-based investor can control the length of the movie, and even determine how the plot goes. Director Ni Kunhao shot the "Defense Line: Secret Escort," an Internet movie about military action, with YOUKU as a major investor in 2020. At that time, this movie also had its competitor "Sniper" (2020), an action war movie directed by Huang Zhaosheng in 2020. In light of the broadcast data provided by the platform, the investor demanded modifications including the film script, requiring a battle scene every five minutes in the film, and the action and battle scenes in the beginning part of the movie to last for at least 10 minutes. The investor also demanded that the proportion of the action and battle scenes take $65 \%$ of the total length of the film, with the non-action part of the movie only serving as the transition between the action parts. The fulfillment of the said demands was taken as the basis for the division of revenue between platforms. Therefore, the big data analysis model that the Internet-based investor relies on not only limits the creative freedom of the creators, but also makes Internet big movies gradually become similar in theme, subject matter and narrativity, just like the "Pop Art" for mass production. Under a fixed model, Internet big movies have gradually generated a pattern of assembly-line production similar to but more simplified than that of traditional film production. 


\section{Simplified assembly-line production}

Similar to traditional film production, the production of Internet big movies also involves a fine division of labor, and a production crew including: script creator, director, cameraman, lighting engineer and film cutter. These professionals have their own job duties and coordinate with each other. For a traditional theater-chain-based movie, RMB 30 million yuan is only the benchmark of a small and medium investment, while for an Internet big movie, RMB 10 million yuan already reaches the price ceiling. The lower investment makes the composition of the production crew for an Internet big movie more simplified, which thus is unable to guarantee the quality of the Internet big movie. However, in this production model, movies can be made with low cost in a short period of time, allowing more creative individuals to take part in film production.

With the simplified creation model, Internet big movies have brought more opportunities for novices at film creation to practice their skills. The production of Internet big movies has seen leapfrog growth: the total number of Internet big movies released on major video websites increased from around 450 in 2014 to some 700 in 2015, and to 2193 in 2016, a surge more than threefold, which means there were nearly 7 movies released every day during that period ${ }^{[9]}$. In 2018 , the total box office takings in China were 60.71 billion yuan (the actual net box office takings were about 56.6 billion yuan), while the size of the pay-to-watch Internet video market amounted to 53.65 billion yuan in 2018 . From this it can be seen that the size of the pay-to-watch Internet video market is expected to surpass the box office takings. In January 2020, 62 Internet movies were released on various platforms, and 12 films earned more than 10 million, breaking the historical record ${ }^{[10]}$. In recent years, more and more film production teams have engaged in Internet big movie projects, pushing the total number of Internet big movies to an increasingly higher level. However, targeting the lower-end market and produced in line with lower standards, Internet big movies focus more on how to make the audiences excited, following popular topics or types of films to do imitations, so as to achieve a high flow of viewers, rather than looking into the depth in art of the film, which is pursued by theater-chain-based movies. Blindly relying on big data, Internet big movies tend to be made in the same key in terms of content, type and style, losing artistic characteristics and originality. In the production process, traditional film and television centers are converted from "creators" into "audiences and users," to customize movies for the users. When the viewer clicks on the fast-forward button, it suggests that he/she is not interested in this part of the film; when the viewer clicks on the rewind button, it suggests that he/she would like to watch again a wonderful or touching part of the movie; when the viewer clicks on the pause button, it suggests that this part is boring and cannot make the viewer stay firm in his/ her seat ${ }^{[1]}$. To meet the demands suggested by the big data of the market, Internet big movies are prone to be stereotyped and superficial, leading to aesthetic fatigue on the audiences as time passes. In addition, the surging number of Internet big movies would also exacerbate the variation in quality.

\section{Conclusions}

In the prosperous period of classic Hollywood, the highly developed work system of the major industrialized movie studios in Hollywood directly contributed to the birth of genre films. However, "the unrestrained pursuit of profit led films to unilateral development, impeding the creativity of directors, actors and other staff in the industry." ${ }^{[12]}$. "As time goes by, artistic creations gradually became degenerated and devitalized, falling into a dilemma." ${ }^{[13]}$

Today, with the intervention of big data, Internet big movies are made under a production system, to some extent, similar to that of the major movie studios in Hollywood. Internet big movies, though being a type based on the development of the Internet, should also be a popular type with artistic and commercial attributes. However, this type of movie now is dominated by big data and commercial value, being pushed to "unisexual reproduction"- the blindly self-reproduction of genre films, or simple imitations of successful films." ${ }^{[13]}$. In addition to the similar production system, Internet big movies now can only cater to the audience's taste, rather than creating popular taste among the audiences, and the "audiences" are no longer humans but the emotionless data in the context of Internet.

Due to the implementation of the censorship policy according to which films banned on TV are not allowed to be released on Internet, Internet platforms began to self-censor repetitive and vulgar their contents. "In 2018, under the guidance of the policy, mainstream video websites carried out a strict self-inspection and cleaning operation, removing 454 Internet movies from their platforms." ${ }^{[14]}$ In addition to tightened regulation, the increasing demand of the audiences for high-quality content are also in turn pushing Internet big movies to focus aesthetic attributes and quality in creation.

The endless repetition of commercial film types under the advancement of industrial technology cannot promote the healthy development of film art. In the process of rapid development, Chinese Internet big movies have taken many detours, but now have seen gradual upgrade and transformation based on continuous exploration. With the increasingly mature market environment, we expected that Internet big movies would bring more artistic enjoyment to the audiences. 


\section{References}

[1] Hu Xing. The convergent change of losing artistic attributes in Chinese films under the new media environment [J] Home Drama, 2018(01):65-67+70.

[2] Data Source: The release of 47th Statistical Report on China's Internet Development [J]. China Broadcasts, 2021(04):38.

[3] Zhou Yuyuan. The release of 2020 Report on Network Audio-Video Development in China: the interpretation of the status and trends of the industry [J]. China Radio Film and TV, 2020(21):47-51.

[4] Cao Juan and Wang Nan. Analysis of the current situation of the Internet movie industry in 2020 [J]. Movie Literature, 2021(06):24-30.

[5] Data Source: Douban.com. Search the key word "Wojin".

[6] Yang Hui. Multiple Paths to Trans-media Video Production: A study on the category differences and box office performance of IP-based movies in China [J]. Modern Audio-Video Arts, 2020(02):39-43.

[7] Chen Liwen. Opportunities and challenges in the upgrading of China's Internet movie industry [J]. Media Forum, 2021, 4(20):73-74.

[8] Zhu Lin. The Internet-based dissemination of Chinese movies and the innovative development of Internet Movies. [J] Chinese Film Market, 2021(05):36-40.

[9] Data source: China Film Industry Report 2017.

[10] Data source: Report on the operation of Chinese film market in 2019 and development trend in 2020, iiMedia.

[11] Pan Yuefeng. Advantages and Disadvantages of Using Big Data in TV drama Production - a discussion on the gains and losses of Netflix using big data and its enlightenment [J]. Journal of Guilin Normal College, 2017, 31(03):68-71.

[12] Yang Yuanying. Introduction to film. [M]. Beijing. Beijing United Publishing Company, 2017.2:288.

[13] Hao Jianzhu. Genre film tutorial. [M]. Shanghai. Fudan University Press, 2011.8:22-24,379.

[14] Lu Jiajia and Liu Hanwen. Analysis report on the development of Chinese film industry in 2018 [J]. Contemporary Cinema, 2019(03):13-20. 